УДК 519.682.6

\title{
МАТЕМАТИЧЕСКОЕ МОДЕЛИРОВАНИЕ ТРАНСПОРТНОГО ПОТОКА НА ПРИМЕРЕ ЯНДЕКС.ТАКСИ Г. УФА РЕСПУБЛИКИ БАШКОРТОСТАН
}

\author{
Мельник Любовь Юрьевна \\ к.ф.-м.н., доцент \\ Улыбин Владислав Сергеевич \\ студент \\ ФГБОУ ВО «Уфимский государственный \\ нефтяной технический университет»
}

\begin{abstract}
Аннотация: в статье рассматривается математическое моделирование транспортного потока Яндекс.Такси в программном пакете Matlab.Simulink.

Ключевые слова: математическая модель, транспортный поток, дифференциальное уравнение, численное решение.

\section{MATHEMATICAL MODELING OF TRAFFIC FLOW ON THE EXAMPLE OF YANDEX.TAXI, UFA REPUBLIC OF BASHKORTOSTAN}

Melnik Lyubov Yurievna Ulybin Vladislav Sergeevich

\begin{abstract}
The article discusses the mathematical modeling of the Yandex.Taxi traffic flow in the Matlab.Simulink software package.

Key words: mathematical model, traffic flow, differential equation, numerical solution.

Заторы на дороге - одна из наиболее важных, глобальных проблем современного мира. Чрезвычайно быстрое развитие, повсеместное использование автотранспорта и стихийно растущий спрос на автомобили среди всех слоев населения заставляет тысячи проектировщиков и инженеров дорожно-транспортных служб ежедневно работать над улучшением транспортных сообщений, однако предусмотреть все - задача невозможная, и нередки случаи, когда на дороге случается перегрузка, что
\end{abstract}


впоследствии ведет к образованию затора [1, с. 32]. Чтобы понимать причины и возможные последствия затора, необходимо знать, как он появляется, поэтому построим математическую модель, описывающую образование затора, на примере работы Яндекс.Такси г. Уфа Республики Башкортостан.

Для составления математической модели введем ряд допущений:

1. По ширине одной полосы может находиться только один автомобиль.

2. Участники дорожного движения соблюдают правила дорожного движения (ПДД), то есть начинают движение, когда загорается зеленый сигнал светофора, и останавливаются, когда загорается красный.

3. Затор не изменяется скачкообразно, то есть затор не может возникнуть мгновенно, точно так же, как исчезнуть [2, с. 14].

Пусть имеется некоторая функция $\varphi(t)$, именуемая транспортным потоком, также введем параметры, характеризующие конкретную дорогу, автостраду, улицу или проспект. Одним из таких параметров будет являться пропускная способность $N$. Пропускная способность $N$ обратно пропорциональна эффективной дорожной ширине. В свою очередь, эффективную дорожную ширину будем представлять в виде произведения количества полос дороги на ширину одной полосы.

Введем параметр $L$, характеризующий протяженность участка дороги. В качестве участка дороги можно рассматривать расстояние от одного светофора до другого. Пусть протяженность участка дороги $L$ прямо пропорциональна пропускной способности дороги $N$,

$$
N=\frac{L}{k \cdot l}
$$

Наконец, для согласования размерности пропускной способности введем коэффициент $W$. Коэффициент $W$ характеризует пропускную способность дороги единичной длины, единичной ширины в единицу времени, тогда пропускная способность дороги окончательно будет описываться следующим уравнением:

$$
N=W \cdot \frac{L}{k \cdot l}
$$

Пусть функция $U(t)$ характеризует желаемую интенсивность дорожного потока, тогда произведение транспортного потока на пропускную способность дороги будет равна функции $U(t)$, то есть

$$
U(t)=\varphi(t) N .
$$


Наконец, для создания дифференциального уравнения, описывающего возникновение транспортного затора, введем коэффициент $I$, характеризующий инерционность создания пробки на дороге

$$
U(t)=\varphi(t) \cdot N+I \cdot \frac{d}{d t} \cdot \varphi(t)
$$

Для решения полученного дифференциального уравнения введем нулевые начальные условия, такие, что

$$
\varphi\left(t_{-0}\right)=\varphi\left(t_{+0}\right)=0
$$

Иными словами, нулевые начальные условия дифференциального уравнения постулируют, что транспортный поток не изменяется скачкообразно, то есть затор не может возникнуть мгновенно, точно так же, как исчезнуть, что соответствует третьему пункту допущений. Расчет режима до начала движения (при $\left.t=0_{-}\right): \varphi(t)=0$.

Составим характеристическое уравнение с учетом, что

$$
I \cdot \frac{d}{d t} \cdot \varphi(t) \rightarrow p \cdot I
$$

тогда,

$$
Z(p)=N+p \cdot I=0
$$

следовательно,

$$
p=-\frac{N}{I} \text {. }
$$

Так как получен один действительный отрицательный корень, то переходный процесс будет апериодическим. Запишем свободную составляющую транспортного потока от времени

$$
\varphi_{\mathrm{cB}}(t)=A \cdot e^{p t},
$$

где $A$ - постоянная интегрирования.

Определение постоянной интегрирования по начальным условиям

$$
\varphi_{\text {св }}(t)=\left.A \cdot e^{p t}\right|_{t(0+)}=A \cdot e^{0}=A
$$

таким образом,

$$
\varphi_{c \mathrm{~B}}(0)=A .
$$

Расчет установочного режима после начала движения

$$
\varphi_{\text {пр }}=\frac{U}{N}
$$

Свободную составляющую транспортного потока $t=0_{+}$, найдем как алгебраическую разницу между переходной и принужденной величиной

$$
\varphi_{c \mathrm{~B}}\left(0_{+}\right)=i \cdot\left(0_{+}\right)-\varphi_{\text {пр }}=0-\frac{U}{N}=-\frac{U}{N}
$$

следовательно,

$$
A=-\frac{U}{N}
$$


тогда,

$$
\varphi_{\text {св }}(t)=-\frac{U}{N} \cdot e^{-\frac{N}{I} t}
$$

Транспортный поток· $\varphi(t)$ найдем как сумму принуждённой и свободной составляющих

$$
\varphi(t)=\varphi_{\text {пр }}+\varphi_{\text {св }}(t)=\frac{U}{N}-\frac{U}{N} \cdot e^{-\frac{N}{I} t}=\frac{U}{N}\left[1-e^{-\frac{N}{I} t}\right]
$$

Проверка решения:

$$
\begin{array}{r}
U=\left[\frac{U}{N}-\frac{U}{N} \cdot e^{-\frac{N}{I} t}\right] \cdot N+I \cdot \frac{d}{d t}\left[\frac{U}{N}-\frac{U}{N} e^{-\frac{N}{I} t}\right]=U-U \cdot e^{-\frac{N}{I} t}+\frac{I \cdot U}{N} \cdot \frac{N}{I} \cdot e^{-\frac{N}{I} t}= \\
U-U \cdot e^{-\frac{N}{I} t} \equiv U
\end{array}
$$

То есть решение

$$
\varphi(t)=\frac{U}{N}\left[1-e^{-\frac{N}{I} t}\right]
$$

является истинным.

Построим кривые согласно уравнению (18) в программном комплексе Matlab. На рисунке 1 показано решение дифференциального уравнения (4) при следующих параметрах $I=1, k=2, l=3.5, L=350 \mathrm{м}, W=0,02, U=50$, время моделирования $t=10$ мин. Из полученного решения (18) можно сделать вывод, что фактическое изменение транспортного потока носит экспоненциальный характер. Для решения дифференциального уравнения (4) численными методами используя программный пакет MatlabSimulink, используем те же допущения и нулевые начальные условия (5).

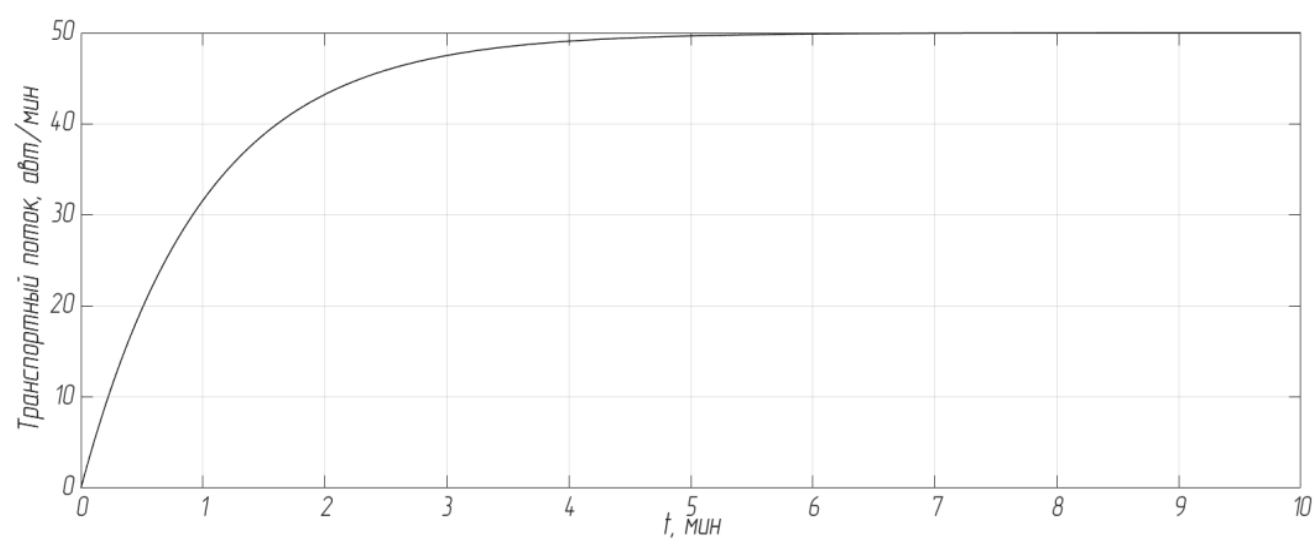

Рис. 1. Решение дифференциального уравнения (4) 
Для того чтобы найти решение дифференциального уравнения, необходимо представить его в виде передаточной функции $W(s)$ в операторной форме. Для этого выполним преобразование Лапласа. Тогда,

$$
\frac{\Phi(s)}{\vartheta(s)}=\frac{1}{N+s \cdot I}=W(s)
$$

Представим дифференциальное уравнение (4) в виде структурной схемы. Структурная схема, описывающая дифференциальное уравнение (4) с начальными условиями (5) приведена на рисунке 2.

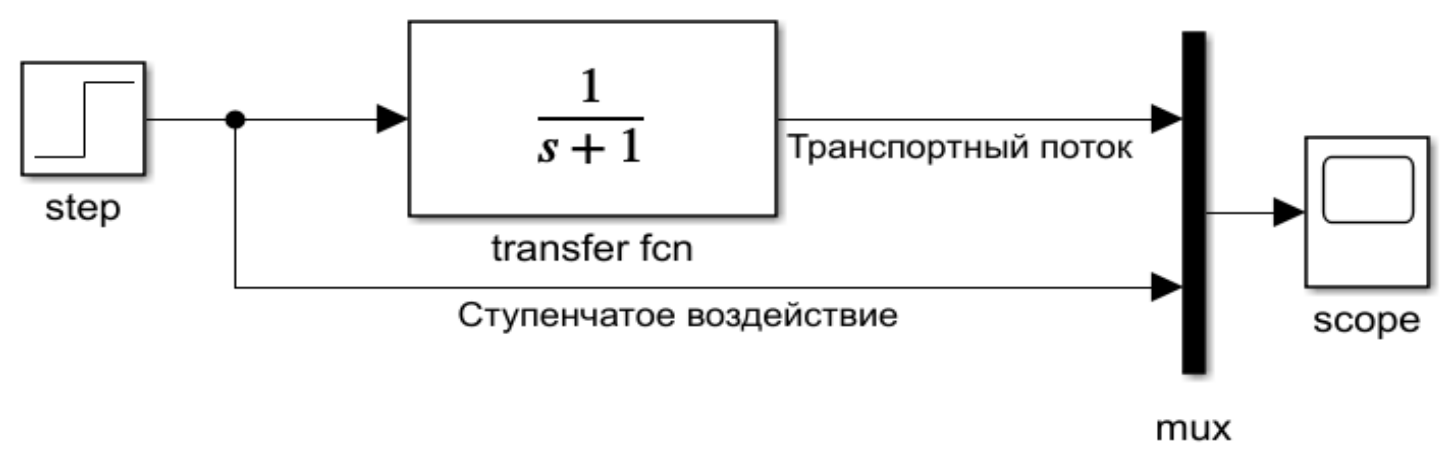

\section{Рис. 2. Структурная схема в программном комплексе MatlabSimulink}

Нетрудно заметить, что полученная функция представляет собой апериодическое звено первого порядка. Для того чтобы графопостроитель scope одновременно отображал ступенчатое воздействие и решение дифференциального уравнения, используется мультиплексирование каналов графопостроителя при помощи элемента тих. Блок step моделирует единичное ступенчатое воздействие Хэвисайда [5]. Ступенчатое воздействие (для наглядности решения) действует в момент времени $t=1$ мин. После выполнения моделирования получим следующую кривую, представленную на рисунке 3 , при следующих параметрах $I=1, k=2, l=3.5, L=350$ м, $W=0,02, U=50$, время моделирования $t=10$ мин. Пунктирной линией показано скачкообразное изменение желаемой интенсивности транспортного потока, основной линией показано фактическое изменение транспортного потока $\varphi(t)$. 


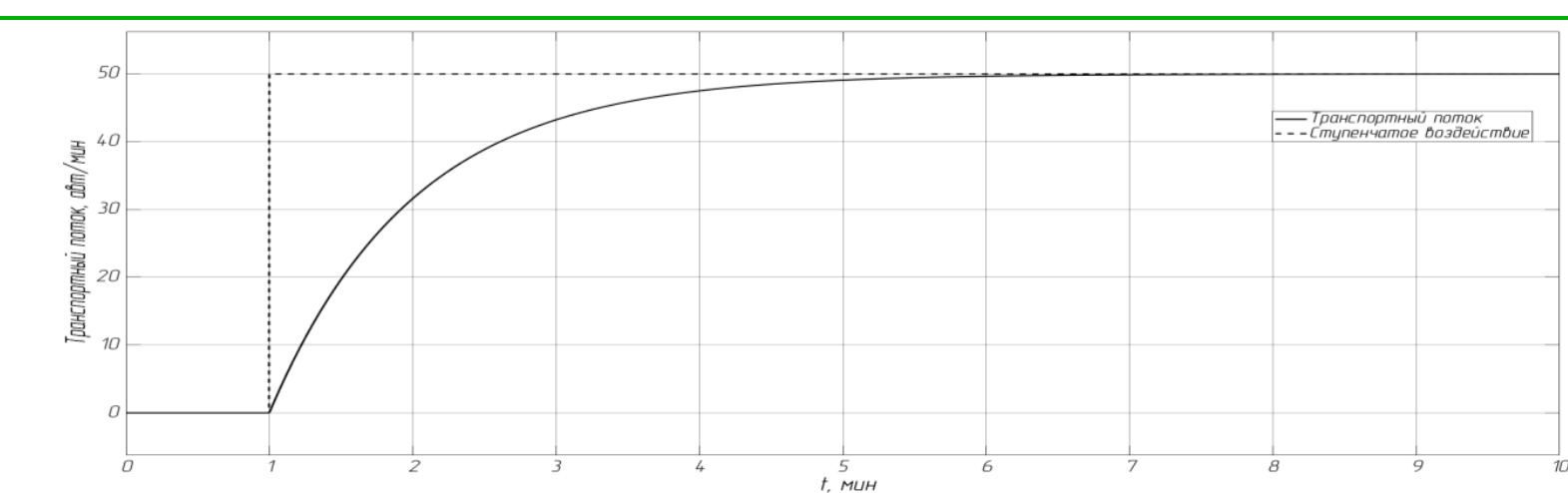

Рис. 3. Численное решение дифференциального уравнения (4)

Из полученного решения можно сделать вывод, что фактическое изменение транспортного потока носит экспоненциальный характер, что подтверждает правильность решения дифференциального уравнения (4) аналитическим методом. Проведем численный анализ при следующих условиях: пусть пропускная способность дороги не изменяется

$N=$ const $=1$, а коэффициент, характеризующий инерционность создания пробки, будет принимать следующие значения $I_{1}=1 ; I_{2}=0,5 ; I_{3}=1,5$. На рисунке 3 показано численное решение дифференциального уравнения (4), при значениях коэффициента инерционности создания затора $I_{1}<I_{2}<I_{3}$

Анализируя рисунок 4, можно сделать вывод, что чем меньше значение коэффициента $I$, тем круче экспоненциальная функция изменения транспортного потока, таким образом, заторы создаются меньше на тех дорогах, на которых значение коэффициента $I$ минимально.

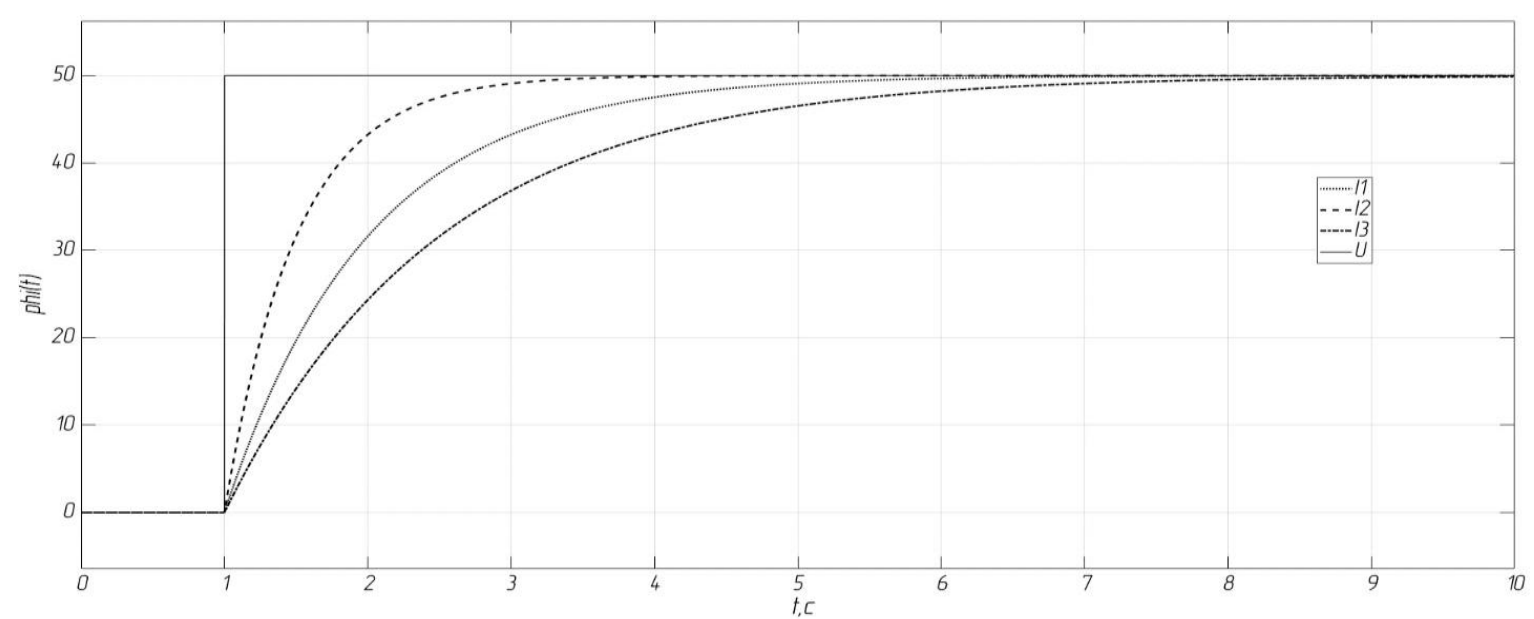

Рис. 4. Численное решение дифференциального уравнения (1) при значениях коэффициента инерционности создания затора $I_{1}<I_{2}<I_{3}$ 
Проведем исследование для ситуации, когда коэффициент создания затора $I=$ const $=1$, а пропускная способность дорог $N_{l}=1 ; N_{2}=0,5 ; N_{3}=1,5$. На рисунке 5 показано численное решение дифференциального уравнения (4) при различных значениях коэффициента пропускной способности.

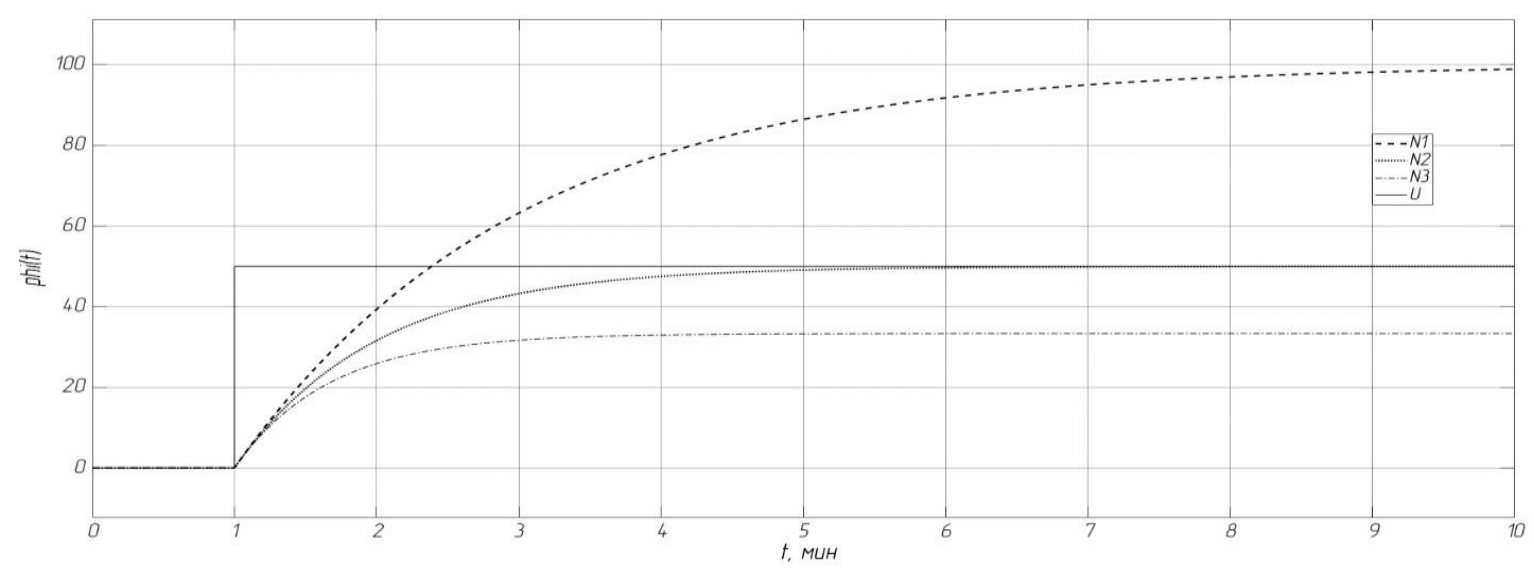

Рис. 5. Численное решение уравнения (4) при различных значениях пропускной способности дороги $N_{1}<N_{2}<N_{3}$

Анализируя рисунок 5, можно сделать вывод, что чем больше пропускная способность дороги, тем больше установившееся значение транспортного потока, то есть дорога, имеющая большую пропускную способность, способна удовлетворять большему значению желаемой интенсивности движения.

Таким образом, полученную математическую модель образования заторов можно аппроксимировать на всю транспортную систему города, оперируя понятиями эквивалентной пропускной способности и эквивалентным коэффициентом инерционности создания заторов.

Согласно статистическим данным распределение транспортного потока во временной области носит нелинейный, дискретный характер, представленный на рисунке 6

Анализируя рисунок 6, можно сделать вывод о том, что транспортный поток в г. Уфа Республики Башкортостан резко увеличивается в утренние и вечерние часы, причем вечерний пик более затяжной, чем утренний. 


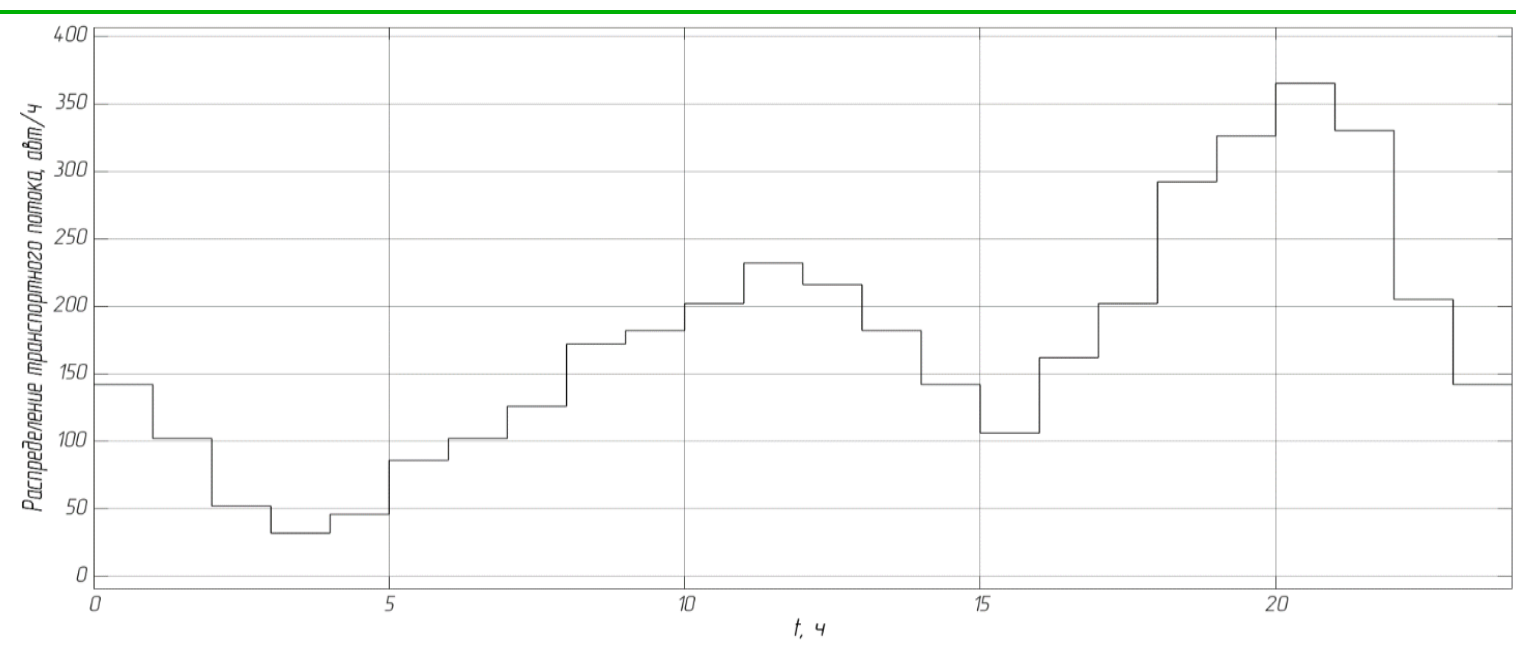

Рис. 6. Распределение транспортного потока во временной области

Рассмотрим поведение эквивалентной структурной схемы автомобильной транспортной сети города с учетом нелинейной дискретной функции распределения транспортного потока в течение суток. На рисунке 7 показан результат моделирования эквивалентной транспортной сети города.

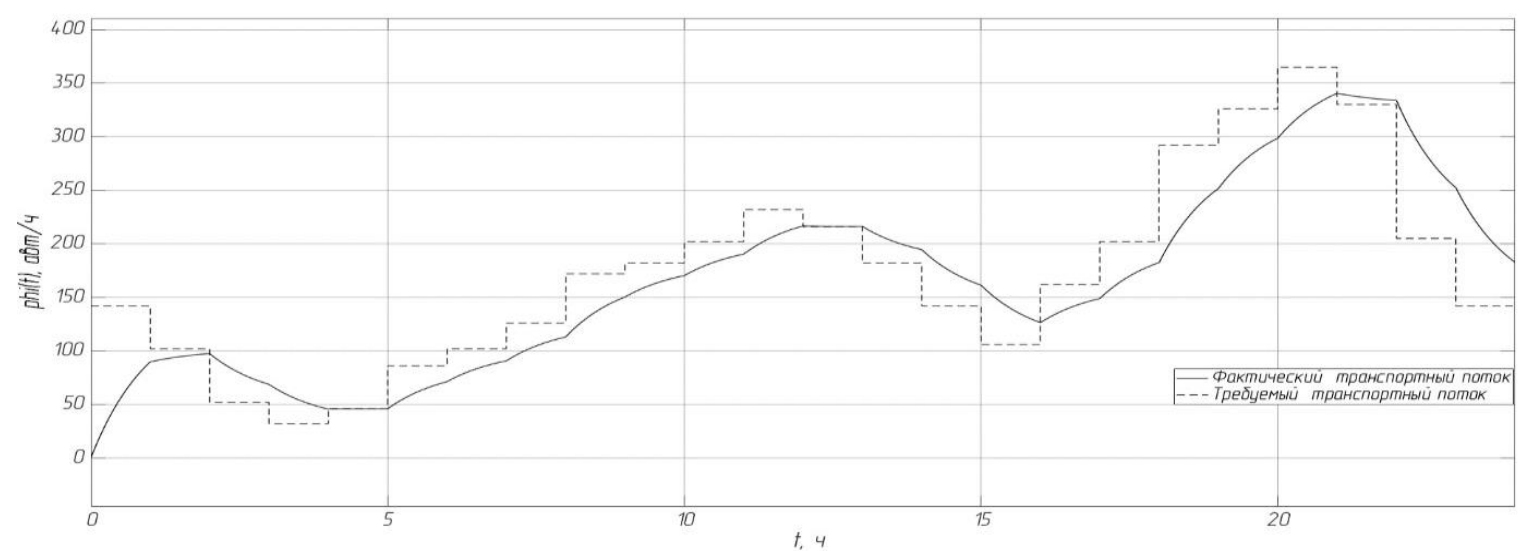

Рис. 7. Распределение транспортного потока по эквивалентной схеме транспортной сети города

Из рисунка 7 видно, что заторы в основном образуются в вечерние часы. Для устранения данной проблемы необходимо изменять либо пропускную способность, либо коэффициент создания затора.

Таким образом, полученная математическая модель четко описывает процесс формирования заторов на дороге [3, с.15]. Математическая модель также позволяет проводить оценку интенсивности движения на определённом участке транспортной системы города и района. Благодаря изменению коэффициентов $N$, I можно моделировать отдельные дорожные ситуации. Имеются возможности по расширению модели до: модели оптимального 
выбора маршрута следования по времязатратному критерию, критерию финансово затратной оценки; модели работы пассажиро/грузоперевозок логических компаний; модели работы компаний, занимавшихся проектировкой транспортных сетей.

\section{Список литературы}

1. Афонин А.М., Транспортная логистика: организация перевозки грузов: Учебное пособие. - М.: Форум, 2017. - 336 с.

2. Введение в математическое моделирование транспортных потоков / Под ред. А.В. Гасникова и др. - М.: МЦНМО, 2015. - 26-36 с.

3. Шапкин А.С. Математические методы и модели исследования операций: Учебник. - М.: Дашков и К, 2017. - 400 с.

(с) Л.Ю. Мельник, В.С. Улыбин, 2021 